\title{
Modal Control in Semiconductor Optical Waveguides With Uniaxially Patterned Layers
}

\author{
Arsen V. Subashiev and Serge Luryi, Fellow, IEEE
}

\begin{abstract}
Uniaxially patterned (UAP) dielectric layers have an optical anisotropy that can be externally controlled. This paper examines the effects of patterning the cladding or the core layer of a three-layer optical waveguide on the polarization properties of propagating radiation. Particular attention is paid to the case when the core material is a semiconductor with optical gain. A number of devices are discussed based on incorporating a UAP layer in the structure design, such as a polarization-insensitive amplifier, a polarizer, an optically controlled polarization switch, and an optically controlled modal coupler.
\end{abstract}

Index Terms-Dielectric waveguides, directional couplers, optical polarizers, photonic crystals, semiconductor lasers.

\section{INTRODUCTION}

$\mathbf{S}$ TRUCTURES with cylindrical air pores forming a twodimensional (2-D) periodic lattice in a semiconductor material are actively studied for photonic bandgap applications [1]-[3], such as spontaneous emission control and light confinement in microcavities. These studies stimulated numerous computations of the photonic crystal (PC) band spectra based on the plane wave expansion of the electromagnetic field [4], [5]. Such calculations showed that in the long wavelength limit the spectrum of electromagnetic waves can be well described in the effective media approximation with an effective dielectric constant corresponding to the results of Maxwell Garnett theory (see, e.g., [6] and [7]). Optical properties of the composite structures patterned with cylindrical holes, for the wavelength $\lambda$ exceeding the interhole spacing $a$, i.e., for $\lambda \gg a$, are described in terms of the filling factor $f$ alone (i.e., the fraction of the total volume occupied by the pores) and do not depend on the long-range order of the holes or their diameter. The effect of disorder is only a weak Rayleigh-like scattering. The effective media approach remains valid for very large contrast ratios between the semiconductor and the pore permittivities [8]-[10] and for arbitrary propagation directions of the electromagnetic waves. Direct comparison of the calculation results based on three-dimensional (3-D) and 2-D modeling shows that the same approach can be used to describe the waveguiding properties of

Manuscript received May 3, 2005; revised September 29, 2005. This work was supported by the New York State Center for Advanced Sensor Technology (Sensor CAT) at Stony Brook.

A. V. Subashiev is with the Department of Electrical and Computer Engineering, State University of New York, Stony Brook, NY 11794-2350 USA, on leave from the State Polytechnic University, St. Petersburg 195251, Russia (e-mail: subashiev@ece.sunysb.edu).

S. Luryi is with the Department of Electrical and Computer Engineering, State University of New York, Stony Brook, NY 11794-2350 USA (e-mail: Serge.Luryi@stonybrook.edu).

Digital Object Identifier 10.1109/JLT.2005.863280 multilayered structures that include patterned layers. Moreover, studies of PC-like structures with a small disorder showed that the Maxwell Garnett approach remains valid even when the requirement $\lambda \gg a$ is relaxed to $\lambda>a$, so long as the optical frequency is below the lowest photonic bandgap and light scattering remains negligible (a similar situation prevails in electronic spectra engineering with quantum well and superlattice heterostructures).

In this paper, we explore the variable anisotropic optical properties of uniaxially patterned (UAP) layers and find that they can be useful in the design of numerous optical devices that are sensitive to the shape and polarization of the optical mode, such as polarizers, lasers, amplifiers, and modulators. The UAP layer anisotropy is not accompanied by any additional optical loss and therefore can be used effectively for the modal control of optical emitters and amplifiers.

Polarization sensitivity is an important factor in semiconductor lasers and amplifiers. It depends on the modal gain that in turn depends on both the material gain anisotropy and the mode confinement factor [11]. The traditional three-layer waveguide design of semiconductor amplifiers with isotropic constituents leads to a better confinement of the TE mode and a larger gain for this mode compared to the TM mode [12], [13]. To obtain a polarization-insensitive amplifier, one had to use highly anisotropic active layers with the material gain that favors TM polarization. Adoption of UAP media for the waveguide layers gives an additional possibility to compensate for the difference in TE-TM confinement, which is inherent to the isotropic situation.

Possible applications of the waveguide structures with a UAP layer extend to the territory already tested experimentally for PC layers, such as structures with a periodically patterned cladding layer, e.g., [2], and a periodically patterned active layer, e.g., [14] and [15]. The relative value of modal propagation constants can be altered by varying the thickness of the core region or the fill factor of the patterned layer. The propagation constants can be further fine tuned by changing the optical contrast between the waveguide constituents with an applied field or optical pumping. Tuning effects are enhanced in structures that are particularly sensitive to the anisotropy of each layer, such as asymmetric waveguides with a thin core layer.

\section{Dielectric Function OF UAP LAYERS AND WAVEGUIDE MODES}

Anticipating a broad scope of possible applications, we consider a three-layer waveguide in which all three layers [the top 


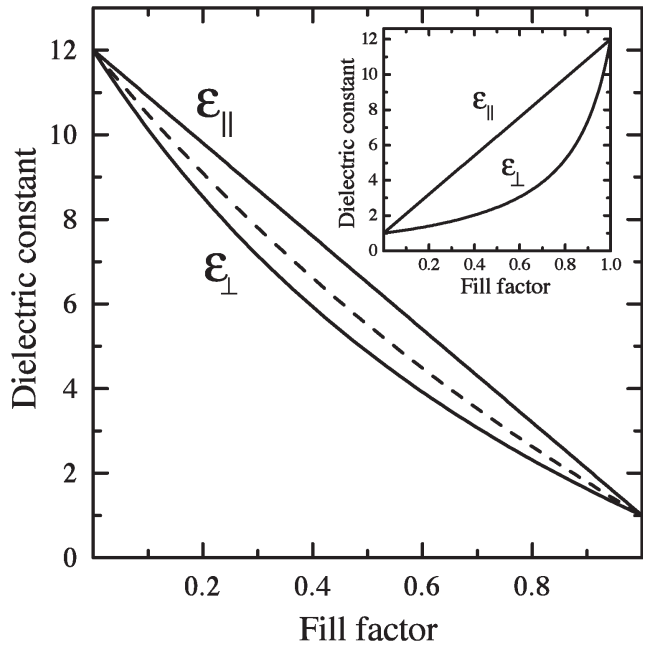

Fig. 1. Average permittivities $\epsilon_{\|}$and $\epsilon_{\perp}$ of a silicon $\left(\epsilon_{\text {out }}=12\right)$ layer UAP with cylindrical air pores $\left(\epsilon_{\mathrm{in}}=1\right)$ for $\mathbf{E} \| C$ and $\mathbf{E} \perp C$, respectively. The dotted line shows the average refractive index (squared). The inset shows the $\epsilon_{\|}$and $\epsilon_{\perp}$ for a structure of dielectric cylinders $\left(\epsilon_{\text {in }} \rightleftarrows \epsilon_{\text {out }}\right)$.

cladding $(c)$, the guiding core $(g)$, and the substrate or bottom cladding $(s)$ ] may be UAP. We assume a thin core layer (of thickness $d$ ) that can support only the lowest propagation modes. We examine the case when the optical axis $C$ of the patterned layers is perpendicular to the waveguide plane. We denote by $\epsilon_{\|, c, g, s}$ and $\epsilon_{\perp, c, g, s}$ the permittivities of the $c, g$, or $s$ layers for two directions of the electric field: parallel $(\|)$ and perpendicular $(\perp)$ to the optical axis, respectively. The permittivity of the inhomogeneous medium in a long-wavelength limit is obtained in the Maxwell Garnett approximation.

For $s$-polarization $(\mathbf{E} \| C)$, the permittivity of a 2-D array of infinitely long cylinders is obtained by direct averaging, viz.,

$$
\epsilon_{\|}=\epsilon_{\text {out }}+\left(\epsilon_{\text {in }}-\epsilon_{\text {out }}\right) f
$$

where the permittivities $\epsilon_{\text {in }}$ and $\epsilon_{\text {out }}$ are inside and outside the cylinder, respectively.

For $p$-polarization $(\mathbf{E} \perp C)$, permittivity is given by

$$
\epsilon_{\perp}=\epsilon_{\text {out }} \frac{\left(\epsilon_{\text {in }}+\epsilon_{\text {out }}\right)+\left(\epsilon_{\text {in }}-\epsilon_{\text {out }}\right) f}{\left(\epsilon_{\text {in }}+\epsilon_{\text {out }}\right)-\left(\epsilon_{\text {in }}-\epsilon_{\text {out }}\right) f} .
$$

Modern derivation of (2) in the long-wave limit of the lowest branch spectrum of the 2-D PC formed by a periodic set of dielectric cylinders can be found in [6]. As shown in [7], the validity of (2) relies on the dipole approximation for the local field between the cylinders. The approximation is also correct for nonperiodic distributions of cylinders, provided $\left(\epsilon_{\text {in }}-\epsilon_{\text {out }}\right) f,\left(\epsilon_{\text {in }}-\epsilon_{\text {out }}\right)(1-f) \ll 1$, and is found to be a very good approximation so long as the cylinder distribution is sufficiently homogeneous $(f \leq 0.5)$, and the index contrast ratio is less than about 40 .

The dependence of $\epsilon_{\|}$and $\epsilon_{\perp}$ on the filling factor is shown in Fig. 1 for the case of cylinder pores in a dielectric medium, with $\epsilon_{\text {in }}=1$ and $\epsilon_{\text {out }}=12$. The anisotropy of refractive index is evidently not small, e.g., for $f=0.3$, one has $\left(n_{\|}-n_{\perp}\right) /\langle n\rangle=0.1$. Also shown is the value of permittivity that would correspond to an average refractive index, i.e.,

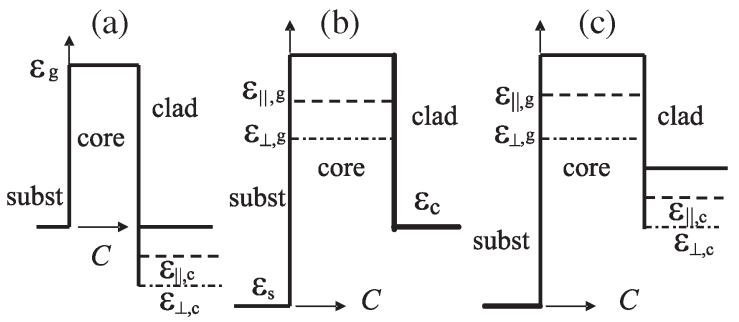

Fig. 2. Profile of the dielectric function in the three-layer waveguide with PL layers: (a) Patterned cladding layer; (b) patterned core layer; and (c) patterned core and cladding layers.

$\langle n\rangle=n_{\text {in }} f+n_{\text {out }}(1-f)$, which is sometimes used; see, e.g., [16], without justification. ${ }^{1}$

The inset in Fig. 1 shows the permittivities $\epsilon_{\|}$and $\epsilon_{\perp}$ for a "mirror" array of cylindrical rods in air. This geometry offers a substantially higher optical anisotropy. Mathematically, it is described by (1) and (2) with the replacement $\epsilon_{\text {out }} \rightleftarrows \epsilon_{\text {in }}$.

Note that (2) fails for thin UAP layers when the height of the cylinders becomes comparable to their diameter. For this case, the effective media approach remains valid, but (2) must be modified to allow for depolarization factors of the finite-height cylinders.

Below, we discuss the properties of a three-layer waveguide. We shall employ the usual approach [11] developed for isotropic waveguide constituents. The guided modes supported by the structure will be calculated using the values $n_{\|}$and $n_{\perp}$ as polarization-dependent refractive indices of the patterned layers. Exemplary profiles of the dielectric function are shown in Fig. 2 for a waveguide with patterned cladding layer (a), an asymmetric waveguide with a UAP core (b), and a hypothetical structure with both cladding and core layers patterned (c).

\section{A. TE Mode}

In this mode, the electric field is perpendicular to the pore axes, so that propagating waves are ordinary in all three layers. Hence, to find the modal index, we can use the eigenvalue equation for the propagation wave vector in an isotropic layered structure [11]

$$
k_{o, g} d=\tan ^{-1}\left(\frac{\alpha^{c}}{k_{o, g}}\right)+\tan ^{-1}\left(\frac{\alpha^{s}}{k_{o, g}}\right)
$$

where the substitution $\epsilon_{c, g, s} \rightarrow \epsilon_{\perp, c, g, s}$ is made, viz., $k_{o, g}=$ $\sqrt{\epsilon_{\perp, g} k_{0}^{2}-Q^{2}}, \quad \alpha^{c}=\sqrt{Q^{2}-\epsilon_{\perp, c} k_{0}^{2}}, \quad \alpha^{s}=\sqrt{Q^{2}-\epsilon_{\perp, s} k_{0}^{2}}$, $k_{0}=\omega / c$, and $Q$ is the propagation wave vector, which defines the mode effective index $n_{\text {eff }}=Q / k_{0}$.

The cutoff thickness $d_{c, \mathrm{TE}}$ of the active layer for the case $\epsilon_{\perp, s}>\epsilon_{\perp, c}$ is found from (3) as

$$
d_{c, \mathrm{TE}}=\left[k_{0} \sqrt{\left(\epsilon_{\perp, g}-\epsilon_{\perp, s}\right)}\right]^{-1} \tan ^{-1}\left(\sqrt{a_{\mathrm{TE}}}\right)
$$

\footnotetext{
${ }^{1}$ We note that in the case considered $\left(\epsilon_{\text {in }} \ll \epsilon_{\text {out }}\right)$, the "average" index $\langle n\rangle$ is rather close to $n_{\perp}$. However, in the opposite case of $\epsilon_{\text {in }} \gg \epsilon_{\text {out }}$ (dielectric cylinders in air), the value of $\langle n\rangle$ is intermediate between the true refractive indices for the two polarizations and differs strongly from both.
} 
where $a_{\mathrm{TE}}$ is an asymmetry parameter of the form

$$
a_{\mathrm{TE}}=\frac{\epsilon_{\perp, s}-\epsilon_{\perp, c}}{\epsilon_{\perp, g}-\epsilon_{\perp, s}}
$$

The case $\epsilon_{\perp, s}<\epsilon_{\perp, c}$ is described by replacing $\epsilon_{\perp, s} \rightleftarrows \epsilon_{\perp, c}$.

In the limit of a thin active layer $k_{o, g} d \ll 1$, (3) yields

$$
n_{\mathrm{eff}, \mathrm{TE}}=\sqrt{\epsilon_{\perp, s}}+\frac{\delta_{\mathrm{TE}}^{2}}{2 \sqrt{\epsilon_{\perp, s}}}
$$

where

$$
\delta_{\mathrm{TE}}=\frac{k_{0}^{2} d^{2}\left(\epsilon_{\perp, g}-\epsilon_{\perp, s}\right)^{2}-\left(\epsilon_{\perp, s}-\epsilon_{\perp, c}\right)}{2 k_{0} d\left(\epsilon_{\perp, g}-\epsilon_{\perp, s}\right)} \ll 1 .
$$

For an amplifying structure with active core, the gain factor for the TE mode $G_{\mathrm{TE}}$ can be calculated as [12]

$$
G_{\mathrm{TE}}=-\frac{k_{0}}{n_{\mathrm{eff}, \mathrm{TE}}} \frac{\int_{0}^{d} d x \epsilon_{g}^{\prime \prime}\left|E_{y}\right|^{2}}{\int_{-\infty}^{\infty} d x\left|E_{y}\right|^{2}}
$$

where $\epsilon_{g}^{\prime \prime}$ is an imaginary part of the active layer permittivity, $x$ is taken along $C$, and $y$ is perpendicular to the wave propagation direction. For a UAP core, the integration in (8) includes taking the average over the layer plane. The modal gain (8) can be written as a product of the material gain $G_{g}=k_{0} \epsilon_{g}^{\prime \prime} / \sqrt{\epsilon_{g \text {, out }}}$ and the optical confinement factor $\Gamma_{\mathrm{TE}}$, which can be calculated explicitly in the dipole approximation for the field distribution in the patterned layer. For a structure with a thin patterned core layer, $\Gamma_{\mathrm{TE}}$ equals

$$
\Gamma_{\mathrm{TE}}=F \sqrt{\frac{\epsilon_{g, \text { out }}}{\epsilon_{s}}} \frac{2 \sqrt{\epsilon_{s}-\epsilon_{c}+\delta_{\mathrm{TE}}^{2}}}{\sqrt{\epsilon_{s}-\epsilon_{c}+\delta_{\mathrm{TE}}^{2}}+\delta_{\mathrm{TE}}} \delta_{\mathrm{TE}} k_{0} d
$$

where $F$ is the local field factor $s=\left(1-f+B^{2} f\right) /$ $(1+B f)^{2}$, and $B=\left(\epsilon_{g, \text { out }}-\epsilon_{g, \text { in }}\right) /\left(\epsilon_{g, \text { out }}+\epsilon_{g, \text { in }}\right)$. For a symmetric waveguide, (9) reduces to the well-known result [17]. For an asymmetric waveguide, $\delta_{\mathrm{TE}}$ rapidly decreases with the difference between the indices of the cladding and substrate layers, as follows from (7). This leads to a high sensitivity of the optical confinement to both the asymmetry of the waveguide and the layer anisotropy.

\section{B. TM Mode}

For the TM mode, the electric field has two components: one perpendicular and the other parallel to the $C$ axis. The propagating waves are extraordinary in the UAP layers. The eigenvalue equation is of the form

$$
k_{e, g} d=\tan ^{-1}\left(\frac{\epsilon_{\perp, g} \beta^{c}}{\epsilon_{\perp, c} k_{e, g}}\right)+\tan ^{-1}\left(\frac{\epsilon_{\perp, g} \beta^{s}}{\epsilon_{\perp, s} k_{e, g}}\right)
$$

where $k_{e, g}=\sqrt{\epsilon_{\perp, g} k_{0}^{2}-\left(\epsilon_{\perp, g} / \epsilon_{\|, g}\right) Q^{2}}$

$$
\beta^{c}=\sqrt{\left(\frac{\epsilon_{\perp, g}}{\epsilon_{\|, g}}\right) Q^{2}-\epsilon_{\perp, c} k_{0}^{2}}
$$

and $\beta^{s}=\sqrt{\left(\epsilon_{\perp, g} / \epsilon_{\|, g}\right) Q^{2}-\epsilon_{\perp, s} k_{0}^{2}}$. The cutoff thickness $d_{c, \mathrm{TM}}$ for a structure with $\epsilon_{\|, s}>\epsilon_{\|, c}$ is given by

$$
d_{c, \mathrm{TM}}=\left[k_{0} \sqrt{\left(\frac{\epsilon_{\perp, g}}{\epsilon_{\|, g}}\right)\left(\epsilon_{\|, g}-\epsilon_{\|, s}\right)}\right]^{-1} \tan ^{-1}\left(\sqrt{a_{\mathrm{TM}}}\right)
$$

where the asymmetry parameter $a_{\mathrm{TM}}$ has the form

$$
a_{\mathrm{TM}}=\frac{\epsilon_{\perp, g} \epsilon_{\|, g}\left(\epsilon_{\|, s}-\epsilon_{\|, c}\right)}{\epsilon_{\perp, c} \epsilon_{\|, c}\left(\epsilon_{\|, g}-\epsilon_{\|, s}\right)} .
$$

The case $\epsilon_{\|, s}<\epsilon_{\|, c}$ is described by replacing $\epsilon_{\|, s} \rightleftarrows \epsilon_{\|, c}$ and $\epsilon_{\perp, s} \rightleftarrows \epsilon_{\perp, c}$. For a thin active layer $k_{e, g} d \ll 1$, (10) yields

$$
n_{\mathrm{eff}, \mathrm{TM}}=\sqrt{\epsilon_{\|, s}}+\frac{\delta_{\mathrm{TM}}^{2}}{2 \sqrt{\epsilon_{\|, s}}}
$$

where

$$
\delta_{\mathrm{TM}}=\frac{k_{0}^{2} d^{2} r_{1}^{2}\left(\epsilon_{\|, g}-\epsilon_{\|, s}\right)^{2}-r_{2}^{2}\left(\epsilon_{\|, s}-\epsilon_{\|, c}\right)}{2 k_{0} d r_{1}\left(\epsilon_{\|, g}-\epsilon_{\|, s}\right)}, \quad \delta_{\mathrm{TM}} \ll 1
$$

with $r_{1}=\sqrt{\epsilon_{\|, s} \epsilon_{\perp, s}} / \epsilon_{\|, g}$ and $r_{2}=\sqrt{\epsilon_{\|, s} \epsilon_{\perp, s} / \epsilon_{\|, c} \epsilon_{\perp, c}}$.

In calculations of the modal gain $G_{\mathrm{TM}}$ and the optical confinement $\Gamma_{\mathrm{TM}}$ for the TM mode, one must correctly evaluate the energy flux in and outside the active layer [12]. We write down the optical confinement factor for a structure with a thin patterned core layer

$\Gamma_{\mathrm{TM}}=(1-f) \frac{\epsilon_{s} \sqrt{\epsilon_{s} \epsilon_{g, \text { out }}}}{\epsilon_{\|, g}^{2}} \frac{2 \sqrt{\epsilon_{s}-\epsilon_{c}+\delta_{\mathrm{TM}}^{2}}}{\sqrt{\epsilon_{s}-\epsilon_{c}+\delta_{\mathrm{TM}}^{2}}+\delta_{\mathrm{TM}}} \delta_{\mathrm{TM}} k_{0} d$.

Due to a small multiplier $r_{1}^{2}$ in the numerator of (14), both $\delta_{\mathrm{TM}}$ and, hence, $\Gamma_{\mathrm{TM}}$ are even more sensitive to asymmetry and anisotropy than the analogous parameters for the TE mode. When the active layer is thin, $k_{e, g} d \ll 1$, and the confinement for the TM mode is smaller by a factor of $\approx\left(\epsilon_{s} / \epsilon_{g}\right)^{3}$ than that for the TE mode. For thicker layers, $k_{e, g} d \sim 1$, this ratio reduces to $\left(\epsilon_{s} / \epsilon_{g}\right)^{2}$; see, e.g., [12] and [13].

The mode competition in laser structures is also affected by the difference in the reflection coefficients for the competing modes. $^{2}$ For a cleaved stripe structure, the modal reflection coefficients $R_{m}$ are given by [18]

$$
R_{m}=\frac{\left(n_{\mathrm{eff}, m}-1\right)^{2}}{\left(n_{\mathrm{eff}, m}+1\right)^{2}}, \quad m=\mathrm{TE}, \mathrm{TM}
$$

Thus, in the effective index approach, this ratio is a function of $n_{\text {eff,TE }}$ and $n_{\text {eff,TM }}$ and, therefore, is also affected by the fill factor of the UAP layers of the waveguide.

\footnotetext{
${ }^{2}$ In addition, the difference in the reflection coefficients for the two modes also favors TE mode generation. It is, however, less important for a long enough cavity and can be easily taken into account.
} 

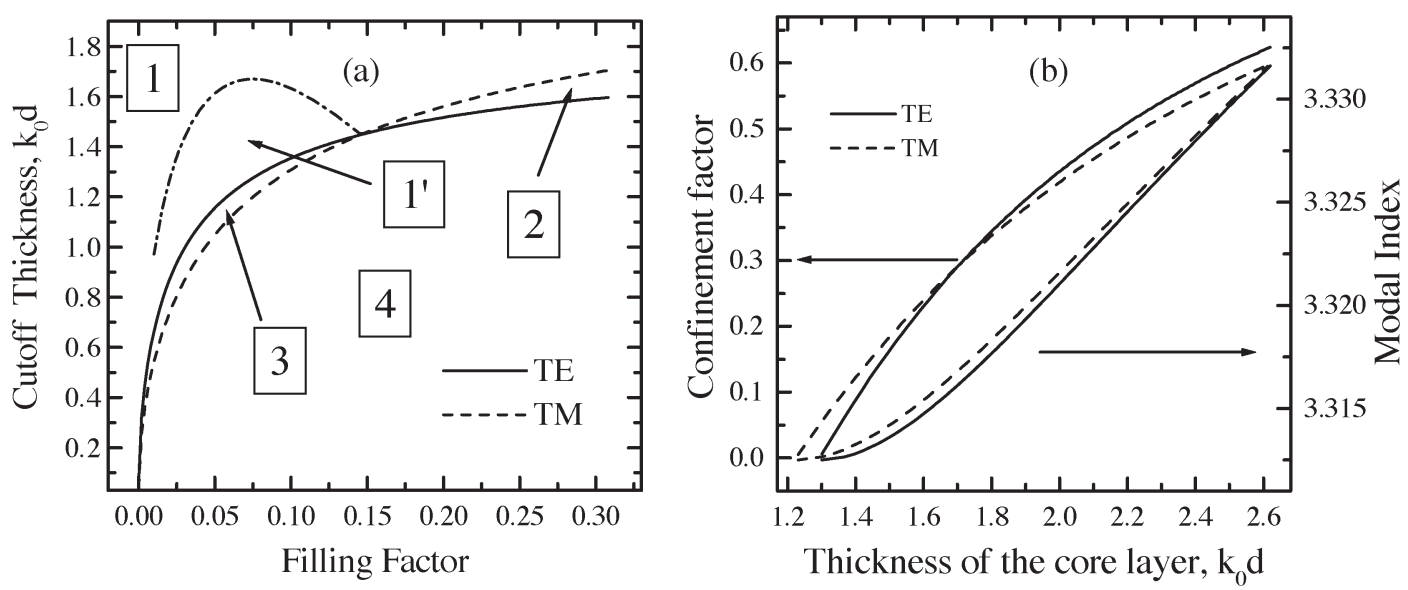

Fig. 3. (a) Cutoff thicknesses for TE and TM modes as functions of the fill factor in the UAP cladding layer for $\mathrm{Al}_{0.17} \mathrm{Ga}_{0.83} \mathrm{As}_{\mathrm{G}} \mathrm{GaAs} \mathrm{Al} \mathrm{l}_{0.17} \mathrm{Ga} \mathrm{g}_{0.83} \mathrm{As}$ structure. Four distinct mode confinement regions are identified: 1) Both modes are confined, 2) only the TE mode is confined, 3) only the TM mode is confined, and 4) both modes are not confined. The dash-dotted line corresponds to the locus of points on the graph, where the confinement of both modes is the same. It delineates a region $\left(1^{\prime}\right)$ within 1), where $\Gamma_{\mathrm{TM}}>\Gamma_{\mathrm{TE}}$. (b) Variation of the optical confinement factors and the modal indices of the two modes with active layer thickness $d$ in a UAP structure as in (a) for a fixed filling factor $f=0.08$. Equal modal gain at $\lambda=860 \mathrm{~nm}$ is achieved for $d=182 \mathrm{~nm}$.

\section{Possible Applications of UAP Structures}

We have shown that the cutoff thicknesses and modal propagation constants in waveguides with a thin core layer are sensitive to the permittivities of the layers and their patterning. Small variations of the propagation constants result in substantial changes of the confinement factor modal ratio. This modal control can be employed in optical devices, such as polarizers and mode-insensitive amplifiers. It is important to realize that the control can be effected rapidly and in real time. For example, optical pumping of the UAP layer within the absorption band of one of its constituent materials will change the optical contrast of the uniaxial pattern and thus modify both the refractive index of the UAP layer and the modal indices of the waveguide. Thus, we can have an ultrafast switch of the modal response in an anisotropy-based cutoff device. Other possible applications are mode-dependent leaky waveguides and directional couplers. With an additional highindex layer, adjacent to one of the cladding layers, the coupling of waveguide modes to this layer will have a strong dependence on the matching of modal propagation constants.

\section{A. Mode Tuning and Polarization-Insensitive Amplifier}

To clarify the effects of a UAP layer on waveguide modal properties, we consider the cutoff thickness of a symmetric three-layer waveguide, in which one of the cladding layers is patterned, cf. the index profile in Fig. 1(a). Exemplary material compositions are taken for a GaAlAs heterostructure, specifically, GaAs core and $\mathrm{Al}_{x} \mathrm{Ga}_{1-x}$ with $x=0.17$ for both cladding and substrate layers. We assume the UAP structure in the cladding layer. The alloy refractive index is taken in the form $n(x)=3.4-0.53 x+0.09 x^{2}[19]$.

Variation of the cutoff thicknesses $d_{c, \mathrm{TE}}$ and $d_{c, \mathrm{TM}}$ with the fill factor is shown in Fig. 3(a) in units of $1 / k_{0}$. Both modes are confined in region 1 , and neither mode is supported in region 4. Region 2 supports only the lowest TE mode and region 3 only the lowest TM mode. For a fill factor $f \leq 0.141$, we see that $d_{c, \mathrm{TE}}<d_{c, \mathrm{TM}}$, and we can have a waveguide that supports only the lowest TM wave. For $\lambda=0.86 \mu \mathrm{m}$ and $f=0.08$, the interval where this is the case is $167 \mathrm{~nm} \leq$ $d \leq 177 \mathrm{~nm}$. Similarly, for $f \geq 0.141$, there is an interval of layer thicknesses in which only the TE mode is confined. The reversal of modal confinement is due to a rapid decrease with $f$ of the cladding layer indices for both polarizations. This leads to a better confinement of the TE mode at large $f$, since in a strongly asymmetric waveguide, anisotropy is of minor importance.

The fact that $d_{c, \mathrm{TE}}<d_{c, \mathrm{TM}}$ in a certain range of fill factors indicates that there is a region of core thicknesses in the same range where both modes are supported, but the TM mode has a tighter optical confinement. This region, designated as $1^{\prime}$, is delineated in Fig. 3(a) by the dash-dotted line. In the vicinity of the dash-dotted line, there is another line where gain is mode insensitive (precise position of this line depends on other factors, such as anisotropy of the material gain and modal dependence of the feedback). This enables us to design a mode-insensitive amplifier without relying on those other factors.

It should be noted that in a waveguide with active (amplifying or absorbing) layers, the waveguiding itself is influenced by gain/damping effects. For structures with a thin core layer, $k_{0} d \ll 1$; these effects, however, are smaller than the indexguiding effects by a factor of $\left(k_{0} d\right)^{2}$ (see Appendix I) and can be safely neglected.

Fig. 3(b) shows the variation of optical confinement factors and effective modal indices as functions of the active layer thickness for an exemplary fill factor $f=0.08$. Equal confinement is obtained at $d=1.62 \times k_{0}^{-1}=221.6 \mathrm{~nm}$ (for $\lambda_{0}=$ $860 \mathrm{~nm}$ ). Since at this thickness $n_{\mathrm{eff}, \mathrm{TE}}<n_{\mathrm{eff}, \mathrm{TM}}$, the design of a mode-insensitive amplifier should also take into account the different modal reflection coefficients, cf., e.g., (16).

Pore spacings $\leq 100 \mathrm{~nm}$ and pore diameters $\leq 30 \mathrm{~nm}$ [14], [15] are demanding but achievable with focused ion beam patterning. Parameters of the structure discussed above are adequately addressed with an approximately triangular lattice 


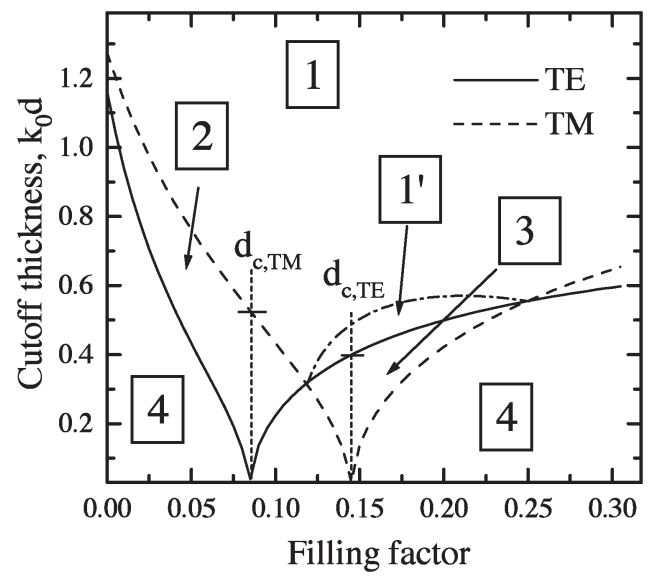

Fig. 4. Cutoff thicknesses for TE and TM modes in an asymmetric structure $\left(\mathrm{Al}_{x} \mathrm{Ga}_{1-x} \mathrm{As} / \mathrm{GaAs} / \mathrm{Al}_{y} \mathrm{Ga}_{1-y} \mathrm{As}\right.$ with $\left.x=0.2, y=0.7\right)$ as functions of the filling factor of the patterned $\mathrm{Al}_{x} \mathrm{Ga}_{1-x}$ As cladding. Vertical lines indicate when the waveguide becomes symmetric for one of the modes. Regions are designated as in Fig. 3.

of pores of diameter $40 \mathrm{~nm}$ and pitch $a=134 \mathrm{~nm}$. For such a lattice, the pitch remains comfortably shorter $(\approx 2$ times $)$ than the wavelength in the media. Requirements to the structure parameters are less demanding in the infrared region.

Equal modal confinement can also be obtained in the conceptually simpler (though probably less practical) case when the UAP layer is the core of a symmetric waveguide. This case can be easily analyzed in a similar fashion. We find that with a thin active layer, the TM mode can be made competitive if one uses a waveguide with a relatively small initial index contrast, which makes it more sensitive to core layer anisotropy. The desired low contrast is obtained by an appropriate choice of the fill factor of the patterned layers.

\section{B. Cutoff Polarizer}

In waveguides based on III-V heterostructures, the index contrast between core and cladding layers is weak. Because of this, the modal competition takes place at small $f$ and for a thin core. The region of competition can be made substantially larger in asymmetric waveguides with properly chosen compositions in the substrate and cladding layers. For the $\mathrm{Al}_{x} \mathrm{Ga}_{1-x} \mathrm{As} / \mathrm{GaAs} / \mathrm{Al}_{y} \mathrm{Ga}_{1-y} \mathrm{As}$ waveguide structure, one should take a smaller Al concentration $x$ in the UAP (cladding) layer than the Al concentration $y$ in the substrate. One can then find the fill factor values $f_{\mathrm{TE}}, f_{\mathrm{TM}}$, for which, respectively, $\epsilon_{c, \perp}=\epsilon_{s}$ and $\epsilon_{c, \|}=\epsilon_{s}$, i.e., the waveguide becomes symmetrical for one of the waves. Fig. 4 shows the variation of cutoff thicknesses for a waveguide structure with $x=0.2$ and $y=0.7$. In the vicinity of $f=0.085$, only the TE mode is confined in the interval of $0 \geq d \geq d_{c, \mathrm{TM}}$, and for $f=0.145$, only the TM mode is confined in the interval of $0 \geq d \geq d_{c, \mathrm{TE}}$. Thus, the waveguide with a judiciously chosen fill factor, and active layer thickness can be used as a cutoff-based polarizer. Moreover, region $\left(1^{\prime}\right)$ can be extended to higher values of $f$ (which would make the structure easier to make) by using a structure with both core and cladding layers patterned.

\section{Dominant Lasing Mode in Highly Asymmetric Structures}

As an example of a highly asymmetric waveguide, we consider a structure with air for the cladding layer and a UAP semiconductor core layer $\left(\mathrm{Si}, \epsilon_{\text {out }}=12\right)$ on a dielectric substrate ( $\mathrm{SiN}, \epsilon_{s}=3.7$ ). The waveguide profile is illustrated in Fig. 2(b).

First, we calculate the cutoff thicknesses for the TE and TM modes. Their dependences on the fill factor of the UAP layer are shown in Fig. 5(a). In the range $f \geq f_{c}=0.53$, the TM mode has a smaller cutoff thickness, and there is a wide range of thicknesses (region 3 ) where the waveguide will support only the lowest TM mode. We see that in strongly asymmetric waveguide structures, at large $f$, the TM mode has better confinement and larger modal index. This results from the faster growth with $f$ of the asymmetry factor $a_{\mathrm{TE}}$ compared to $a_{\mathrm{TM}}$; see (5) and (12). For values of the fill factor near $f_{c}$, both modes have a similar confinement factor in a broad range of $d$, as illustrated in Fig. 5(b). Note that the values of the confinement factors are generally reduced due to the porosity of the core layer. The examined asymmetric waveguide is similar to that used by Cloutier and $\mathrm{Xu}$ [14], who observed a predominantly TM-polarized laser-like emission from a UAP Si-on-insulator layer. The main difference from Fig. 5 is that a lower-index $\mathrm{SiO}_{2}$ was used as the bottom cladding. It would be tempting to seek an explanation for the observed TM polarization in terms of the UAP properties of the waveguide used. However, the structure parameters in [14] correspond to $f=0.18<f_{c}$ and for the stated core layer thickness within region 2 in Fig. 5(a). Not only is the TE mode "better" confined, but the TM mode is not confined at all at the operating point. Therefore, the observed TM polarization of the generated light in the experiment [14] poses a serious problem (see Appendix II).

\section{Polarization Switch}

Under high illumination, the photo-induced concentration of free electrons in the core and/or cladding layer(s) can be large enough for a substantial change of the permittivity and thus effect a change of the modal confinement in a UAP waveguide. Using materials with a short carrier lifetime, both the rise time and the recovery time can be very short, thus providing an ultrafast all-optical modal control. Switching of polarization can be most easily achieved with type- 1 structures, as in Fig. 2(a), when the optical excitation energy is above the absorption edge of the cladding layer but below the absorption edge of the substrate layer. In this case, optical pumping will result in a substantial change of the asymmetry factors for the two modes.

As an example, we consider an asymmetric InGaAsP waveguide [13] operating at $\lambda=1.55 \mu \mathrm{m}$ with core layer index $n=3.55$, substrate layer index $n_{s}=3.24$, and UAP cladding layer index $n_{c}=3.45$. Let the energy of the pump excitation be above the cladding bandgap of $\lambda=1.35 \mu \mathrm{m}$. The resulting variation of waveguiding can be described by taking the dielectric function of the absorbing core and cladding layers with the Drude contribution of free carriers, viz. $\epsilon_{g}=\epsilon_{g, \infty}-\left(\omega_{p, g}^{2} / \omega^{2}\right)$ and $\epsilon_{\text {out }_{c}}=\epsilon_{c, \infty}-\left(\omega_{p, c}^{2} / \omega^{2}\right)$, where 

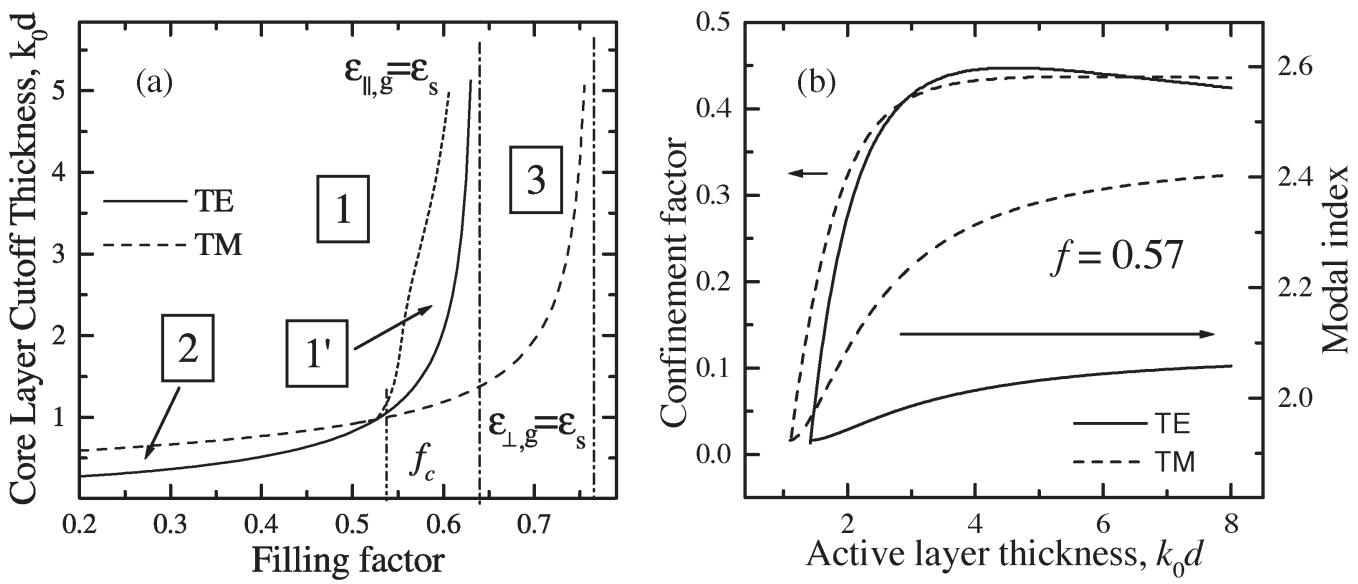

Fig. 5. (a) Cutoff thicknesses and confinement regions for TE and TM modes as a function of fill factor of the UAP core layer for asymmetric Si/SiN waveguide with air as a cladding layer. The regions being noted as in Fig. 3(a). (b) Modal indexes and confinement factors for TE and TM modes as a function of core layer thickness for a structure with $f=0.45$.

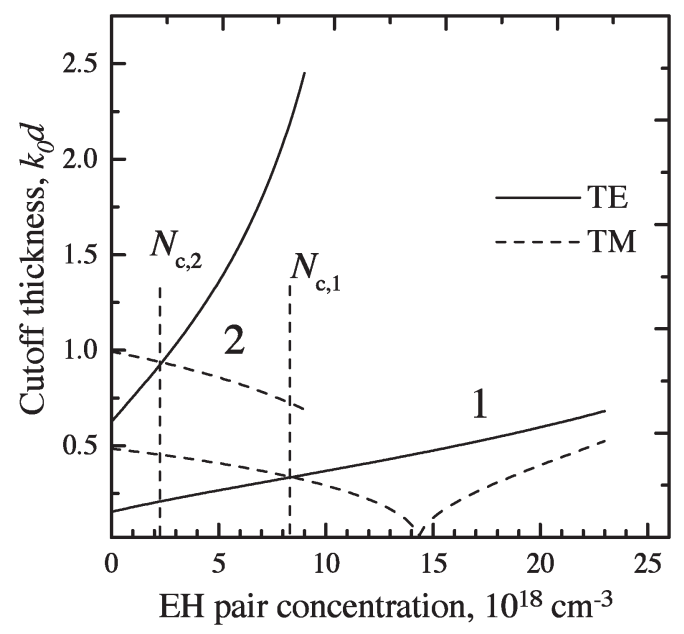

Fig. 6. Cutoff thicknesses for TE and TM modes in an InGaAsP $1.55-\mu \mathrm{m}$ waveguide structure as functions of the concentration of optically pumped electron-hole pairs. Case (1): UAP cladding layer only. Case (2): Both cladding and core layers patterned.

$\omega_{p, i}^{2}=N_{i} e^{2} / \epsilon_{0} m_{i}^{*}$ with $\omega_{p, i}, N_{i}$, and $m_{i}^{*}$ being, respectively, the plasma frequency, the electron-hole pair density, and the reduced effective mass in the core $(i=g)$ and cladding $(i=c)$ layers. The bulk optical dielectric constants of the core and cladding materials are denoted, respectively, by $\epsilon_{g, \infty}$ and $\epsilon_{c, \infty}$.

A linear decrease of the dielectric function of the core and cladding layers with the free carrier concentration alters the waveguiding properties for the two modes. The variation of the cutoff thicknesses is shown in Fig. 6 (case 1). The TM and TE lines intersect at $k_{0} d=0.33$. This means that if we choose the core thickness $d$ to be precisely $d=0.33 / k_{0}$, we shall have only one mode confined for any pumping level. At the pumping corresponding to $N \geq N_{c, 1} \approx 8.5 \times 10^{18} \mathrm{~cm}^{-3}$, the device mode will switch from TM to TE. This effect can be used for both polarization switching and modulation.

The switching concentration $N_{c, 1}$ is sensitive to layer indices and can be adjusted to lower values. For the purpose of low- power switching, a more favorable structure is type-3, with both cladding and core layers patterned as in Fig. 2(c). The cutoff thicknesses for this case are displayed in Fig. 6 (case 2). Making UAP both the core and the cladding adds anisotropy to the structure, and the free carrier effect on wave propagation becomes sharper. This lowers the switching concentration $N_{c, 2}$.

It should be noted that the excitation levels needed to achieve high electronic concentration may lead to heating that changes the refractive indices of the layers. Such thermal effects my become important and should be controlled; these effects can be distinguished by a slower time response [20].

The other effect of high electronic concentration is a build up of the imaginary part of dielectric constant of the layers. This effect is only a small correction (see Appendix I) when index guiding is operative.

Finally, we note that if only the core layer were patterned [as in Fig. 2(b)], the photoinduced free carrier concentration would be insufficient to effect a cutoff-controlled switch between the TE and TM modes as the variation of asymmetry factors would not be strong enough.

\section{Modal Control in Leaky WaVeguides And DiRECTIONAL COUPLERS}

New useful polarization-dependent effects can be obtained when an additional high refractive index layer is added onto the cladding layer or when the three-layer waveguide is placed on a base-substrate layer of high refractive index. These effects have been exploited in the so-called resonant-layer devices [21], vertical directional couplers and filters [22], and leaky waveguides [23]. Uniaxial patterning of one or more waveguide layers can provide a useful addition to the modal control possibilities of these devices. Here, we briefly outline these possibilities.

For waveguides on a high-index base substrate, the main effect of the base substrate results from the exponential decay of the guided modes due to their leakage through the bottom cladding layer into the substrate. This leakage has an exponentially strong dependence on the difference between the modal effective index and the index of the bottom cladding 
layer, which determines the barrier height for photon tunneling decay into the base substrate. In a standard leaky waveguide, the TE mode has a higher index and therefore exponentially lower damping. As follows from our discussion in the previous sections, incorporating a UAP layer in the structure allows us to alter the bottom cladding layer modal transparency. This gives a variable selectivity of the leakage-based modal control.

Adding a high-index resonant layer on the top cladding layer with its thickness chosen to support a mode with the same propagation constant as the basic waveguide leads to an oscillatory energy exchange between the two waveguides. The resonant coupling underlying this exchange is exponentially sensitive to matching of the propagation constants. Incorporation of a UAP layer as a core or a cladding layer, combined with the optical pumping, enables a variable-mode vertical directional coupler that effects fast mode selection at the time of operation.

\section{LATERAL VARIATION OF UAP LAYER PARAMETERS}

Consider the effect of gradual variation in the density of pores in a UAP cladding layer (vary $f$ laterally). In our limit of $\lambda \gg a$ (even relaxed to $\lambda>a$ ), the effect is evidently similar to that of lateral index variation in the cladding. It can be used for shaping the mode field in the laser stripe, to achieve desirable properties, similar to those obtained by the parabolic etching of the stripe or the parabolic variation of the material index. An example of such properties is the one-mode highpower generation in a shaped unstable resonator laser design [24], [25]. It is known that one way of obtaining a large gain difference between the fundamental mode and higher order modes is to use structure profiles with a strong real-index antiguiding and weak imaginary-index guiding. Structures with UAP layers can provide a very effective index antiguiding. In waveguides with a UAP core, one must design the pore density so that it is highest at the center line. On the other hand, in waveguides with a UAP cladding layer, the antiguiding effect is achieved when the density of pores (and hence the index contrast) grows with the distance from the center.

We remark that while UAP layers with lateral variation $f$ are effective for achieving high-power single-mode operation, this approach is suitable only for longer wavelength, e.g., farinfrared devices. One needs room for a smooth but sizable pore density variation while still staying in the limit $\lambda>a$.

\section{CONCLUSION}

We have derived an efficient approach to calculate cutoff thicknesses and optical confinement factors for three-layer semiconductor optical amplifier waveguides with anisotropically patterned layers, in which uniaxial anisotropy is deliberately introduced in one or more of the waveguide layers.

We demonstrate that the patterned layer anisotropy can be effective in providing modal control of useful waveguide devices. Although no attempt was made to optimize the proposed devices, their implementation is shown to be within a reasonable range of lithographic and material parameters.
Finally, we note that the theoretical approach used in this work, based on the effective media approximation in the spirit of the Maxwell Garnett theory, has a wider range of validity than that we have exploited so far. Thus, our approach in Section V can be used to treat 2-D PCs with laterally varying parameters. The scale of lateral variation does not have to be smooth, so long as the spatial scale of the obtained field variation in the structure exceeds the structure pitch. For example, the same approach can be applied to a 2-D PC with an omitted row of pores that could be useful in the implementation of optical routers and splitters.

\section{APPENDIX I}

\section{Guiding EFFECTS IN A Thin WaVEguide With Active (AMPLIFYING OR ABSORBING) LAYERS}

Wave propagation in a three-layer waveguide with active layers has special features: The waves are inhomogeneous in all layers and hence the wave propagation direction is not the same as the local energy propagation direction. Waveguiding is described by a system of equations, the guiding, and the gain/damping effects being interconnected.

We discuss these effects for the case of the TE mode, where we can use the eigenvalue (3) for the propagation wave vector in an isotropic layered structure. The TM mode can be considered similarly.

Consider the case of active layers, when $\epsilon_{g}=\epsilon_{g}^{\prime}+i \epsilon_{g}^{\prime \prime}$. Equation (3) and the equations for $k_{o, g}, \alpha^{c}$, and $\alpha^{s}$ now have both real and imaginary parts and split into pairs, e.g.,

$$
\begin{aligned}
& k_{o, g}^{\prime}=\operatorname{Re} \sqrt{\left(\epsilon_{\perp, g}^{\prime}+i \epsilon_{\perp, g}^{\prime \prime}\right) k_{0}^{2}-\left(Q^{\prime}+i Q^{\prime \prime}\right)^{2}} \\
& k_{o, g}^{\prime \prime}=\operatorname{Im} \sqrt{\left(\epsilon_{\perp, g}^{\prime}+i \epsilon_{\perp, g}^{\prime \prime}\right) k_{0}^{2}-\left(Q^{\prime}+i Q^{\prime \prime}\right)^{2}} .
\end{aligned}
$$

The sign of the imaginary parts of the waves should be taken in the usual manner so that the waves go only out of the layers with a larger gain (or into the layers with larger absorption).

Thus, there are two independent variables $Q^{\prime}, Q^{\prime \prime}$ (the complex propagation constant of the wave) and a system of two equations generated by (3) to find them. The important point is that all waves become inhomogeneous if at least one layer is active: $\epsilon_{i}^{\prime \prime} \neq 0, i=s, c, g$ (excluding the case $\epsilon_{s}^{\prime \prime}=\epsilon_{g}^{\prime \prime}=\epsilon_{c}^{\prime \prime}$ ).

All qualitative features of the waveguiding can be understood in an exemplary case of a thin core layer, $k_{o, g} d \ll 1$, when (3) becomes algebraic, viz.,

$$
k_{o, g}^{2} d=\alpha^{c}+\alpha^{s} .
$$

Let $Q^{2}=k_{0}^{2} z, z=x+i y$. Then, (A.2) reads

$k_{0} d\left(\epsilon_{g}^{\prime}+i \epsilon_{g}^{\prime \prime}-x-i y\right)=\sqrt{x+i y-\epsilon_{c}}+\sqrt{x+i y-\epsilon_{s}}$.

Let us first discuss the simplest case of a symmetric waveguide with $\epsilon_{c}=\epsilon_{s}$. For this case, (A.3) can be solved 
by introducing a dimensionless variable $t=\alpha^{c} / k_{0}=\sqrt{z-\epsilon_{c}}$, for which we have

$$
k_{0} d\left[\delta \epsilon^{\prime}+i \delta \epsilon^{\prime \prime}-t^{\prime 2}-2 i t^{\prime} t^{\prime \prime}+t^{\prime \prime 2}\right]=2\left(t^{\prime}+i t^{\prime \prime}\right)
$$

where $\delta \epsilon=\epsilon_{g}-\epsilon_{c}$. Then, we have

$$
t^{\prime}+\frac{t^{\prime 2} k_{0} d}{2}=\frac{k_{0} d\left(\delta \epsilon^{\prime}+t^{\prime \prime 2}\right)}{2}, \quad t^{\prime \prime}=\frac{k_{0} d \delta \epsilon^{\prime \prime}}{2\left(1+t^{\prime} k_{0} d\right)} .
$$

Several important conclusions follow from (A.5).

First, $t^{\prime \prime} \propto \delta \epsilon^{\prime \prime}$. The imaginary part of $t$ is, essentially, the oscillatory contribution to the exponential decay of the wave outside of the core. So, as is physically obvious, it is proportional to $\delta \epsilon^{\prime \prime}$, that is, to the difference between the net gain (or loss) in the core and the cladding.

Second, the guiding properties of a thin-core waveguide are determined by two parameters $k_{0} d \delta \epsilon^{\prime}$ and $k_{0} d \delta \epsilon^{\prime \prime}$, of which the former defines the usual "effective one-dimensional potential well," and the latter describes the gain (or loss) guiding effects.

Third, we note that the guiding effects of gain or loss are proportional to the square of the gain differences in the core and the cladding, so that layers with step-like absorption are equally good for guiding. However, for a thin core with gain but no index step, the guiding [described by $\operatorname{Re}(t)$ ] is weak, being proportional to the third power of the small parameter $k_{0} d$.

Fourth, we note that even for pure gain variation (i.e., $\delta \epsilon^{\prime}=0$ ), the guiding effect is genuine. Indeed, the core looses energy only to support the growth of the wave in adjacent cladding layers. Inasmuch as the wave amplitude and its energy both decrease exponentially away from the core, the guiding can be viewed as true confinement (hence, we can evaluate the total energy outside the waveguide). Interestingly, the same is true for a lossy core when the profile of the wave is maintained by extracting the energy from adjacent layers.

Finally, for our case of a thin core, the equations can be readily solved perturbatively, that is, by keeping in first approximation only the lowest terms in $k_{0} d$. For $\alpha_{c}$, this yields

$$
\alpha_{c}^{\prime}=\frac{k_{0}^{2} d \delta \epsilon^{\prime}}{2}, \quad \alpha_{c}^{\prime \prime}=\frac{k_{0}^{2} d \delta \epsilon^{\prime \prime}}{2} .
$$

Therefore, in this approximation, there is no gain guiding, but it appears when higher terms in $k_{0} d$ are taken into account. To bring the discussion in closer correspondence to the results in Section II-A, we note that $t$ is identical to $\delta_{\mathrm{TE}}$ [see (7)]. We can use (7) to write down the modal index directly as

$$
n_{\mathrm{eff}, \mathrm{TE}}=\sqrt{\epsilon_{c}+t^{2}} \approx \sqrt{\epsilon_{c}^{\prime}}+\frac{\delta_{\mathrm{TE}}^{2}+i\left[\epsilon_{c}^{\prime \prime}+\frac{\left(k_{0} d\right)^{2} \delta \epsilon^{\prime} \delta \epsilon^{\prime \prime}}{2}\right]}{2 \sqrt{\epsilon_{c}^{\prime}}} .
$$

In connection with (A.7), we make two observations: 1) The second term in the numerator of (A.7) gives the wellknown Dumke result [17] for the confinement factor (the energy confinement has an additional factor of 2), and 2) in a waveguide with thin core and weak confinement, the damping in the cladding is more effective than the gain in the core.

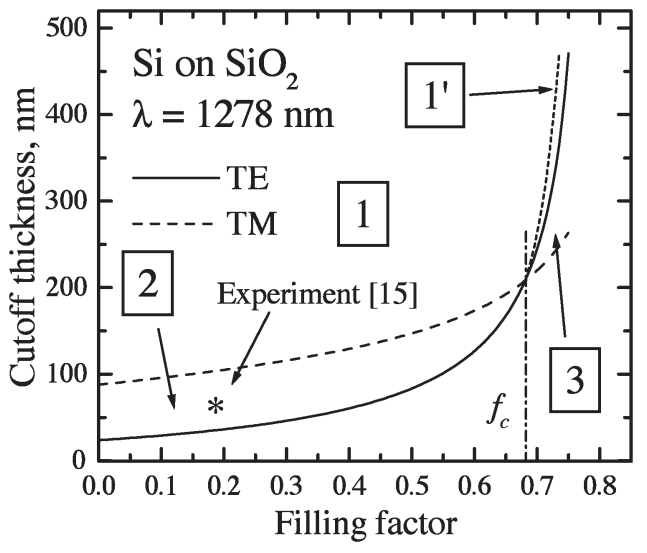

Fig. 7. Cutoff thicknesses for TE and TM modes for a patterned 65-nm-thick $\mathrm{Si}$ layer on $\mathrm{SiO}_{2}$ substrate layer as a function of filling factor. Regions of mode confinement are denoted as in Fig. 3.

For a symmetric waveguide of arbitrary core thickness, the gain guiding effects were recently considered by Siegman [26]. Our analytical treatment in the thin-core limit allows to consider the asymmetric waveguide in a similar fashion.

For the asymmetric case, we retain the definition of the cutoff as the thickness that borders the region where the real part $\operatorname{Re}\left(\alpha^{s}\right)$ of (A.2) vanishes. For a thin core, this cutoff thickness can be found directly from (7), bearing in mind that at the cutoff, $\operatorname{Re}\left(\delta_{\mathrm{TE}}\right)=0$. Damping or gain effects shift the cutoff thickness. This results from the quadratic in $\delta \epsilon^{\prime \prime}$ antiguiding contribution to $\delta_{\mathrm{TE}}$.

An important additional issue in an asymmetric waveguide results from the different signs of the propagative contribution to the $\operatorname{Im}(\delta)$ from the asymmetry factor. Indeed, from (A.4), we have

$$
t=k_{0} d\left(\epsilon_{g}-\epsilon_{s}-t^{2}\right)-\sqrt{\epsilon_{s}-\epsilon_{c}-t^{2}} .
$$

For $k_{0} d \ll 1$, we have $\left|t^{2}\right| \ll\left|\epsilon_{g}-\epsilon_{s}\right|$, so that the lowest order in $k_{0} d$ instead of (7) in the above equation (A.8) gives

$$
\alpha_{c}=k_{0} t, \quad t=k_{0} d\left(\epsilon_{g}-\epsilon_{s}\right)-\sqrt{\epsilon_{s}-\epsilon_{c}} .
$$

Two remarks are in order here. First, we observe that (A.9) coincides with the reduced (7). Indeed, for $t$ to be $\ll 1$, the numerator in (7) should be much smaller than the denominator, so that one can regard the numerator as the difference of two squared and nearly equal terms. Second, we note that we obtain the cutoff thickness for a thin layer with low confinement by setting $\operatorname{Re}(t)=0$ in (A.9).

Separating the imaginary part of (A.9), we obtain

$$
\operatorname{Im}\left(\delta_{\mathrm{TE}}\right)=k_{0} d \operatorname{Im}\left(\epsilon_{g}-\epsilon_{s}\right)-\operatorname{Im}\left(\sqrt{\epsilon_{s}-\epsilon_{c}}\right) .
$$

For a confined mode, the second term in (A.10) is smaller than the first term, but it depends on the difference $\left(\epsilon_{s}-\epsilon_{c}\right)$ and shows the redistribution of loss between the substrate and the cladding. For a weak confinement, the penetration depth into the substrate is larger than that into the cladding, so that substrate contribution prevails. As we decrease $d$, the first term becomes eventually smaller than the second and confinement is lost. 


\section{APPENDIX II \\ EXPERIMENT OF CLOUTIER AND XU [14]}

These authors employed a thin 65-nm UAP Si core layer with a fill factor of $f=0.18$, a thick $\mathrm{SiO}_{2}$ bottom cladding on an $\mathrm{Si}$ substrate, and air for the top cladding. Under optical pumping, they observed light emission at $\lambda=1278 \mathrm{~nm}$ with many characteristics of laser radiation, unpolarized below threshold, and predominantly TM-polarized above the threshold.

The cutoff thicknesses for the two modes at this wavelength are displayed in Fig. 7. We see that except at very high filling factors the TE mode is better confined. Moreover, at the reported parameters of the structure, the TM mode is not confined at all. The reason for this lack of confinement is the strong asymmetry of the air-clad waveguide.

The situation would be rather different if the waveguide was made more symmetric by adding a top cladding layer of refractive index similar to that of the $\mathrm{SiO}_{2}$ bottom cladding layer. In this case, the value of $f_{c}$ would become much smaller and the cutoff thickness for the TM mode would be strongly reduced. This could be enough to shift the structure parameters to regions $1^{\prime}$ or even 3 , where the TM mode is dominant.

The fact that the TE mode looses the competition with TM above threshold may be associated with a mode-selective feedback mechanism, e.g., a higher mirror reflectivity for the TM mode. Another mechanism, which seems more likely to us, may be associated with higher radiative losses for the TE mode due to inhomogeneities of the structure. These inhomogeneities cause (weak) Rayleigh scattering, which does not prevent waveguiding but may damp the resonance mode due to rare but highly efficient long-range fluctuations of the effective index. This mesoscopic mechanism of the damping (to be discussed in a separate publication) is far more effective for TE than for TM modes and depends on the sample size, which can be checked experimentally.

\section{REFERENCES}

[1] E. Yablonovitch, "Inhibited spontaneous emission in solid-state physics and electronics," Phys. Rev. Lett., vol. 58, no. 20, pp. 2059-2062, May 1987.

[2] O. Painter, R. K. Lee, A. Scherer, A. Yariv, J. D. O’Brien, P. D. Dapkus, and I. Kim, "Two-dimensional photonic band-gap defect mode laser," Science, vol. 284, no. 5421, pp. 1819-1821, Jun. 1999.

[3] S. G. Johnson, S. Fan, P. R. Villeneuve, J. D. Joannopoulos, and L. A. Kolodziejski, "Guided modes in photonic crystal slabs," Phys. Rev. B, Condens. Matter, vol. 60, no. 8, pp. 5751-5758, Aug. 1999.

[4] M. Plihal and A. A. Maradudin, "Photonic band structure of twodimensional systems: The triangular lattice," Phys. Rev. B, Condens. Matter, vol. 44, no. 16, pp. 8565-8571, Oct. 1991.

[5] J. D. Joannopoulos, R. D. Meade, and J. N. Winn, Photonic Crystals: Molding the Flow of Light. Princeton, NJ: Princeton Univ. Press, 1995.

[6] N. A. Nicorovici, R. C. McPhedran, and L. C. Botten, "Photonic band gaps: Noncommuting limits and the 'acoustic band'," Phys. Rev. Lett., vol. 75 , no. 8, pp. 1507-1520, Aug. 1995.

[7] A. K. Sarychev and V. M. Shalaev, "Electromagnetic field fluctuations and optical nonlinearities in metal-dielectric composites," Phys. Rep., vol. 335, no. 6, pp. 275-371, Sep. 2000.

[8] F. Genereux, S. W. Leonard, and H. M. van Driel, "Large birefringence in two-dimensional silicon photonic crystals," Phys. Rev. B, Condens. Matter, vol. 63, no. 16, pp. 161101-161105, Apr. 2001.

[9] P. Halevi and F. Ramos-Mendieta, "Tunable photonic crystals with semiconducting constituents," Phys. Rev. Lett., vol. 85, no. 9, pp. 1875-1878, Aug. 2000.
[10] A. A. Krokhin, P. Halevi, and J. Arriaga, "Long-wavelength limit (homogenization) for two-dimensional photonic crystals," Phys. Rev. B, Condens. Matter, vol. 65, no. 11, p. 115 208, Mar. 2002.

[11] M. J. Adams, An Introduction to Optical Waveguides. New York: Wiley, 1981.

[12] T. D. Visser, H. Blok, B. Demeulenaere, and D. Lenstra, "Confinement factors and gain in optical amplifiers," IEEE J. Quantum Electron., vol. 33, no. 10, pp. 1763-1766, Oct. 1997.

[13] Y.-Z. Huang, Z. Pan, and R.-H. Wu, "Analysis of optical confinement in semiconductor lasers," J. Appl. Phys., vol. 79, no. 8, pp. 3827-3830, 1996.

[14] C. Cloutier and J. Xu, Laser-Like Emission From a Periodic All-Silicon Nanostructure, 2004. arxiv: cond-matt/0412376.

[15] X. Wu, A. Yamilov, X. Liu, S. Li, V. P. Dravid, R. P. H. Chang, and H. Cao, Ultraviolet Photonic Crystal Laser, 2004. arxiv: physics/ 0406005 .

[16] M. D. B. Charton, S. W. Roberts, and G. J. Parker, "Guided mode analysis and fabrication of a 2-dimensional visible photonic band structure confined within a planar semiconductor waveguide," Mater. Sci. Eng. B, vol. 49, no. 2, pp. 155-165, Sep. 1998.

[17] H. C. Casey and M. B. Panish, Heterostructure Lasers; Part A: Fundamental Principles. New York: Academic, 1978, pp. 54-57.

[18] J. Buus, "Analytical approximation for the reflectivity of DH lasers," IEEE J. Quantum Electron., vol. QE-17, no. 12, pp. 2256-2257, Dec. 1981.

[19] M. Levinshtein, S. Rumyantsev, and M. Shur, Handbook Series on Semiconductor Parameters, vol. 1. London, U.K.: World Scientific, 1999.

[20] S. W. Leonard, H. M. van Driel, J. Schilling, and R. B. Wehrspohn, "Ultrafast band-edge tuning of a two-dimensional silicon photonic crystal via free-carrier injection," Phys. Rev. B, Condens. Matter, vol. 66, no. 16, pp. 161102-161104, Oct. 2002.

[21] J. M. Hammer, G. A. Evans, G. Ozgur, and J. K. Butler, "Isolators, polarizers, and other optical waveguide devices using a resonant-layer effect," J. Lightw. Technol., vol. 22, no. 7, pp. 1754-1763, Jul. 2004.

[22] K. J. Ebeling, Integrated Opto-Electronics. Berlin, Germany: SpringerVerlag, 1993, pp. 143-170.

[23] L. Torner, F. Canal, and J. Hernandez-Marco, "Leaky modes in multilayer uniaxial optical waveguides," Appl. Opt., vol. 29, no. 18, pp. 2805-2814, Jun. 1990.

[24] S. Guel-Sandoval, A. H. Paxton, S. T. Srinivasan, S. Sun, S. D. Hersee, M. S. Allen, C. E. Moeller, D. J. Gallant, G. C. Dente, and J. G. McInerney, "Novel high-power and coherent semiconductor laser with a shaped unstable resonator," Appl. Phys. Lett., vol. 66, no. 16, pp. 20482050, Apr. 1995.

[25] A. K. Chan, C. P. Lai, and H. F. Taylor, "Antiguiding index profiles in broad strip semiconductor lasers for high-power, single-mode operation," IEEE J. Quantum Electron., vol. 24, no. 3, pp. 489-495, Mar. 1988.

[26] A. E. Siegman, "Propagating modes in gain-guided optical fibers," J. Opt. Soc. Amer. A, Opt. Image Sci., vol. 20, no. 8, pp. 1617-1628, Aug. 2003.

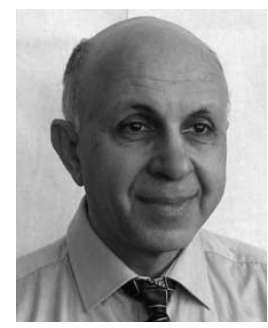

Arsen V. Subashiev received the M.Sc. degree in optoelectronics from St. Petersburg State Polytechnic University, St. Petersburg, Russia, the Ph.D. degree in physics and mathematics from Ioffe Physico-Technical Institute, St. Petersburg, in 1969, and the Doctor of Sciences degree in physics and mathematics from State Polytechnic University, St. Petersburg, Russia, in 1984.

He worked at Ioffe until 1978, when he joined St. Petersburg State Polytechnic University, where he serves as a Professor at the Department of Experimental Physics. He is currently a Visiting Professor in the Department of Electrical and Computer Engineering, State University of New York at Stony Brook, on leave from the St. Petersburg State Polytechnic University. He has published over 90 papers on the theory of electric, photoelectric, and optical phenomena in semiconductors and semiconductor devices. His research interests include the theory of semiconductors and semiconductor nanostructures, theory of spin-polarized electron emission, optoelectronics, and photonics.

Dr. Subashiev served as a member of the Editorial Board and the Executive Editor of Semiconductors, which is a Russian journal distributed in English by American Institute of Physics (AIP), from 1994 to 2004. 


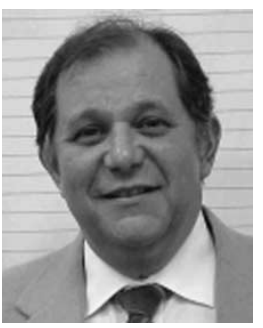

Serge Luryi (M'81-SM'85-F'89) received the $\mathrm{Ph} . \mathrm{D}$. degree in physics from the University of Toronto, Toronto, ON, Canada, in 1978. His doctoral thesis was devoted to theoretical studies of intermolecular interactions in solid hydrogen.

In 1980, he joined Bell Laboratories, Murray Hill, NJ, and became interested in the physics and technology of semiconductor devices. In 1994, he joined the State University of New York at Stony Brook, where he currently chairs the Electrical and Computer Engineering Department. He is also the Founding Director of the NY State Center for Advanced Sensor Technology (Sensor CAT). He has published over 200 scientific papers and holds 42 U.S. patents.

Dr. Luryi was elected Fellow of the IEEE for contributions in the field of heterojunction devices in 1989. He was elected Fellow of the American Physical Society in 1993 for contributions to the theory of electron transport in lowdimensional systems and invention of novel electron devices. He served as the Editor of IEEE TRANSACTIONS ON ELECTRON DEVICES from 1986 to 1990. In 1995, he organized an advanced research workshop on the "Future Trends in Microelectronics: Reflections on the Road to Nanotechnology," which grew into a regular series. The fifth FTM workshop is scheduled for June 2006 (complete information can be found at www.ee.sunysb.edu/serge/FTM.html). In 2003, he was appointed to the rank of Distinguished Professor by the Board of Trustees of State University of New York. He received the Distinguished Member of Technical Staff award from Bell Laboratories in 1990. 